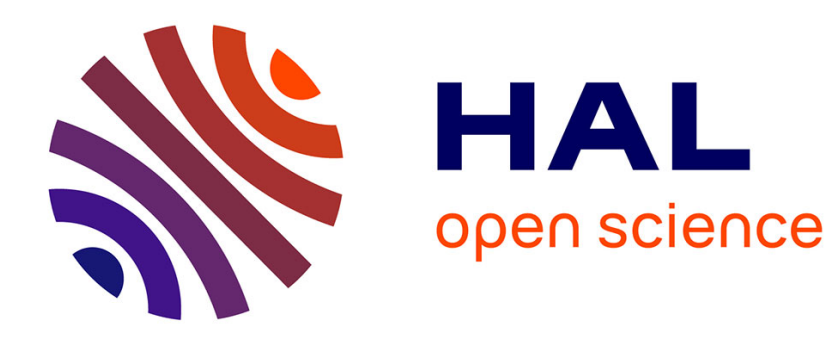

\title{
Le concept de l'insight en psychiatrie
}

N. Jaafari, I. Marková

\section{To cite this version:}

N. Jaafari, I. Marková. Le concept de l'insight en psychiatrie. Annales Médico-Psychologiques, Revue Psychiatrique, 2011, 169 (7), pp.409. 10.1016/j.amp.2011.06.018 . hal-00789287

\section{HAL Id: hal-00789287 \\ https://hal.science/hal-00789287}

Submitted on 18 Feb 2013

HAL is a multi-disciplinary open access archive for the deposit and dissemination of scientific research documents, whether they are published or not. The documents may come from teaching and research institutions in France or abroad, or from public or private research centers.
L'archive ouverte pluridisciplinaire HAL, est destinée au dépôt et à la diffusion de documents scientifiques de niveau recherche, publiés ou non, émanant des établissements d'enseignement et de recherche français ou étrangers, des laboratoires publics ou privés. 


\section{Accepted Manuscript}

Title: Le concept de l'insight en psychiatrie

Authors: N. Jaafari, I. Marková

PII:

S0003-4487(11)00177-6

DOI: doi:10.1016/j.amp.2011.06.018

Reference: $\quad$ AMEPSY 1359

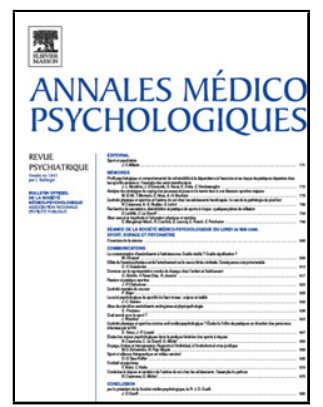

To appear in: $\quad$ Annales Médico-Psychologiques

Please cite this article as: Jaafari N, Marková I, pour Insight Study Goup, Le concept de l'insight en psychiatrie, Annales medio-psychologiques (2010), doi:10.1016/j.amp.2011.06.018

This is a PDF file of an unedited manuscript that has been accepted for publication. As a service to our customers we are providing this early version of the manuscript. The manuscript will undergo copyediting, typesetting, and review of the resulting proof before it is published in its final form. Please note that during the production process errors may be discovered which could affect the content, and all legal disclaimers that apply to the journal pertain. 


\section{Communication}

\section{Le concept de l'insight en psychiatrie}

The concept of insight in psychiatry

\section{N. Jaafari ${ }^{\text {a,b }}$, I. Marková ${ }^{\text {a,c }}$, pour Insight Study Goup}

${ }^{a}$ Unité de recherche clinique intersectorielle en psychiatrie $d u$

Centre Hospitalier Henri Laborit, Poitiers, France

${ }^{b}$ INSERM U 802, CHU et université de médecine et de pharmacie, 86000 Poitiers, France

${ }^{c}$ Department of Psychiatry, University of Hull, Hull, UK

Auteur correspondant: Nematollah Jaafari, Pavillon Toulouse, BP 587, Unité de recherche clinique intersectorielle du Centre Hospitalier Henri Laborit, 86021 Poitiers, France

Adresse email : nemat.jaafari@ch-poitiers.fr

«Il est illusoire de croire que les études empiriques arrivent à cerner le concept de l'Insight avec un grand I, car l'Insight avec un grand I est un concept relationnel qui s'étudie dans la singularité de la relation à l'autre »

\section{Résumé}

C’est en France (particulièrement à la Société Médico-Psychologique de 1869 à 1870), vers le milieu du XIX ${ }^{\mathrm{e}}$ siècle, que le concept d'insight dans les troubles mentaux est apparu. Parmi d’autres idées, l'évolution des opinions concernant la nature de l'aliénation mentale, en particulier l'émergence de la notion de folie partielle, a conduit à des débats sur la question de l'insight chez les patients souffrant d'une maladie mentale. Un regain d'intérêt pour l'insight a été à l'origine de nombreuses études empiriques en psychiatrie pour explorer la relation entre l'insight des patients et les variables cliniques. Les résultats de ces études sont variables et contradictoires. Les explications sont en rapport avec la complexité inhérente du concept de l'insight. Dans l'objectif de clarifier ces divergences, il est essentiel de faire une distinction entre le concept et le phénomène de l'insight et de considérer l'insight comme un concept relationnel. Clarifier ces questions au sujet de l’insight permet de définir les modalités et les limites dans lesquelles il est possible de capturer l'insight de manière empirique. En outre, il facilite la compréhension de l'insight comme un état mental dont les fluctuations dépendent d'une interaction entre des facteurs psychologiques, pathologiques et des facteurs socioculturels. 
Mots clés : Insight ; Maladie mentale ; Psychiatrie

\begin{abstract}
It was in France (particularly in the Société Médico-Psychologique 1869 to 1870) that the concept of insight into mental disorder began to appear around the middle of the $19^{\text {th }}$ century. Amongst other things, changing views concerning the nature of insanity, particularly the emergence of the notion of partial insanity, led to debates about the question of insight in patients with mental illness. Following a resurgence of interest in insight in psychiatry numerous empirical studies have sought to explore the relationship between patients' insight and clinical variables. Results however have been variable and contradictory. Explanations for this can be found in the complexities inherent to the concept of insight. In order to tease these out, it is essential to (i) distinguish between the concept and the phenomenon of insight and (ii) to view insight as a relational concept. Clarifying these issues around insight helps to define the ways and limits in which it is possible to capture insight empirically. In addition, it facilitates an understanding of insight as a mental state whose fluctuations are dependent on an interplay of psychological, pathological and wider sociocultural factors.
\end{abstract}

Keywords: Insight; Mental illness; Psychiatry

\title{
1. Introduction
}

L’insight est un terme anglais qui existe dans plusieurs langues, par exemple les langues anglo-saxonnes, mais n’existe pas dans la langue française ou les langues latines. Il est traduit en français de manière approximative tantôt par " conscience du trouble ", tantôt par « introspection », « déni », « anosognosie ». Ces traductions dépendent du contexte dans lequel l’insight est utilisé, à savoir : "reconnaissance », " compréhension », " raison », « capacité », « jugement », " l’intelligence ». La signification de ces termes est différente selon les disciplines, « déni » en psychanalyse, « la découverte soudaine de la solution d'un problème » en Gestalthérapie, «la capacité cognitive » et «théorie de l'esprit » en psychologie cognitive, l’anosognosie en neuropsychologie, ne représente pas la même chose. Par exemple, l'anosognosie, introduite par Babinski, renvoie à une absence de connaissance 
de son hémicorps controlatéral à la lésion cérébrale alors que le déni et/ou l’introspection renvoient à des processus inconscients avec une connotation psychodynamique.

Cette question de traduction n'est pas seulement un effet de genre ou de traduction, mais elle est fondamentale car de nos jours le terme « insight » est de plus en plus utilisé dans la littérature française sans que l'on comprenne à quoi cela renvoie. Demandez à vos collègues de définir l'insight et vous verrez que chacun a une définition et une traduction et donc une compréhension différente des autres. Ce terme n'est pas propre à la langue française, mais il est commun à toutes les langues latines dans lesquelles le terme insight n’existe pas. Cependant, son application en recherche clinique et dans la prise en charge des patients est d'importance capitale. L'étude de l’OMS en 1973, en utilisant une définition catégorielle de l'insight, rapporte que $70 \%$ des patients présentant un diagnostic de schizophrénie avaient un faible niveau de la conscience de la maladie [in 17]. Cette affirmation n’a pas été validée par Amador et al. en 1994 lors d'une étude de suivi chez 421 patients. Effectivement, ces auteurs, en utilisant une définition multidimensionnelle de l'insight, ont rapporté que le niveau d'insight varie selon les patients : seuls 32,1\% des patients présentaient un faible insight, $25,3 \%$ un insight modéré et $40,7 \%$ un bon niveau d'insight [23].

Effectivement, depuis une vingtaine d'années les études empiriques sur l'insight se sont multipliées [7,18]. Cette recherche a notamment porté en psychiatrie sur la schizophrénie, les troubles bipolaires, les troubles anxieux et les troubles alimentaires. En neurologie, elle s’est portée sur la démence, le traumatisme crânien et l'accident vasculaire cérébral. L’objectif de ces études était de répondre à deux groupes de questions. D’une part, quel est l'intérêt du concept d'insight ? Quel est l'impact de la qualité de l'insight sur le pronostic de la maladie ? Et d'autre part, quels sont les mécanismes qui structurent l'insight ? L’insight est-il intrinsèque à la maladie mentale ? Dans quelle mesure s’agit-il d'une réaction individuelle au trouble mental ? Est-ce un mécanisme adaptatif, protecteur? Quels sont les facteurs externes (environnement, culture...) qui influencent l'insight et dans quelles proportions ?

Afin de répondre à ces questions, de nombreuses études ont tenté de comparer l'insight avec diverses variables comme, par exemple, le pronostic de la maladie, la sévérité de la psychopathologie, les antécédents psychiatriques, la catégorie diagnostique, la compliance au traitement, les variations de l'humeur, les troubles cognitifs, la qualité de vie et le fonctionnement psychosocial, etc. Ces études donnent des résultats divergents et contradictoires dans les troubles psychiatriques comme dans les atteintes neurologiques (pour 
une revue complète, lire Insight en psychiatrie [17]). Les raisons de ces résultats contradictoires sont multiples et seul un retour vers une étape conceptuelle permettrait d'en apporter quelques explications. Cependant, un rappel historique s’impose pour comprendre la naissance et le développement de ce concept.

\section{Un peu d'histoire}

Dans les civilisations occidentales, le concept de l'insight, en tant que phénomène indépendant à explorer et à mesurer, semble s’être développé vers le milieu du XIX ${ }^{\mathrm{e}}$ siècle. Le climat intellectuel de l'époque explique en partie l'essor de ce concept. Effectivement, la pensée philosophique du XVIII ${ }^{\mathrm{e}}$ et du XIX ${ }^{\mathrm{e}}$ siècles se recentre sur l'individu, ce qui permet d'avoir une certaine réflexion sur le Soi et sur les états mentaux subjectifs. Cette réflexion permet d'appréhender différemment la connaissance du Soi et donc son aspect pathologique qui est nommé « la folie ». On assiste dès lors à un intérêt grandissant de la société et de la médecine pour cette notion, avec une augmentation du nombre de structure asilaire, des méthodes d'exploration, des propositions thérapeutiques. Le point fondamental, c'est que cet intérêt pour les pathologies mentales favorise le questionnement sur la conscience de la maladie. Parmi différents facteurs, l'émergence de la notion de «folie partielle » a été particulièrement importante dans les débats portant sur l'insight des patients. La folie partielle visée à décrire la lucidité, la rationalité qui pouvait exister dans « la folie » et sa variabilité dans le temps. Plusieurs écrits démontrent la naissance et l'évolution de ce concept en France. D’abord, les aliénistes français, particulièrement Pinel, rapportent des cas cliniques montrant que les patients conservent une certaine compréhension de leur maladie malgré un raisonnement altéré. En même temps, la justice pose la question de la responsabilité des malades ayant commis des actes criminels. Cette responsabilité n’est possible que si certains aspects de la psyché ne sont pas affectés ; or le concept de la «folie partielle » permettait d'envisager cette possibilité. Puis, les nouveaux courants psychologiques et philosophiques abandonnent la conception d'une psyché indivisible pour une psyché composée d'unités pouvant fonctionner indépendamment. Enfin la phrénologie, elle aussi, plaide pour l'existence de fonctions distinctes localisées dans différentes parties du cerveau. Toutes ces approches ont permis le développement du concept de la « folie partielle », ce concept permettant à son tour l'éclosion du concept d' « insight» [17].

La description du concept de la folie partielle par les aliénistes français et les divergences de l'époque, concernant la nature et les éléments des processus mentaux (raison, 
jugement, sentiment, conscience, etc.), influencent la description des différents aspects du phénomène d'insight. D’un point de vue clinique, l'insight est alors décrit comme un aspect de la maladie mentale (Krafft-Ebing, 1893), exploré par des études cliniques (Billod, 1870, Morel, 1870), utilisé comme un critère pour catégoriser la maladie (Baillarger, 1853 ; Delasiauve, 1861 ; Ritti, 1879), et considéré comme une variable pronostique (Billod, 1870) [17].

En France, plusieurs séances de la Société Médico-Psychologique de 1869 à 1870 ont été consacrées aux «aliénés » qui avaient conscience de leur état. Les discussions autour de ce concept étaient vives, car elles impliquaient la responsabilité médico-légale des patients, comme en peuvent témoigner les articles issus des séances (nous vous en conseillons la lecture, accessible gratuitement sur le site gallica (http://www2.biusante.parisdescartes.fr/livanc/?cote=90152\&do=livr\&fille=o\&cotemere $=901$ $52)$.

Dans l'ensemble, on peut retenir que la considération de la nature de l'insight vers la fin du XIX ${ }^{\mathrm{e}}$ siècle, se fait vers deux concepts opposés. Un concept étroit définissant l’insight comme la conscience des opérations mentales (Despine, 1875 ; Maudsley, 1895) et un concept plus large incluant des jugements plus complexes du patient sur l'impact de la maladie mentale (Dagonet, 1881 ; Billod, 1882 ; Pick, 1882 ; Parant, 1888) [17].

Au début du $\mathrm{Xx}^{\mathrm{e}}$ siècle, la conceptualisation de l’insight se poursuit et s'élargit, mais il subsiste une grande hétérogénéité des descriptions des différents aspects du "phénomène d'insight ». Par exemple, en Allemagne on parle de « Krankheitsbewusstsein » (conscience de la maladie), «Krankheitsgefühl » (sentiment d'être malade), et «Krankheitseinsicht » (conscience incluant des jugements plus complexes sur la maladie), d'autres classifications encore sont définies par Pick (1882), Arndt (1905) [in 15], Aschaffenburg (1915) et Jaspers (1948) [in 17]. Jaspers, lui, distingue d'un côté la conscience d'un changement (krankheitsbewusstsein) et, de l'autre, les jugements portés sur ce changement (krankheitseinsicht). Un effort particulier est porté pour tenter de comprendre les mécanismes qui influencent l'insight (l’intelligence, la culture, le vécu, la mémoire) (Arndt, 1905 ; Jaspers, 1913) [in 17]. En 1934 Lewis affirme la nécessité pour décrire la conscience de se référer au sujet sain, et développer des mesures de concordance entre le patient et le clinicien [in 6]. Les travaux sur l'insight seront fortement diminués avec la Seconde Guerre mondiale et l'aprèsguerre : les chercheurs se sont plus intéressés aux travaux sur la psychiatrie biologique que sur la conceptualisation de l'insight. Il faudra attendre les années 1980 pour voir apparaître des travaux de réflexion sur l’insight. 
De nos jours, l'existence d'un nombre important de définitions et d'échelles d'évaluation de l'insight explique en partie les résultats contradictoires retrouvés à partir des études empiriques. Dans un souci de clarté, nous proposons de visiter l'insight à travers trois notions, l'insight clinique, l'insight cognitif, l'insight somatosensoriel.

\section{Différentes considérations de l’insight}

\subsection{Insight clinique}

L’insight clinique peut être défini comme l'aspect de l'insight relatif à la conscience de la maladie et son implication en termes d'une prise en charge thérapeutique. Dans l'objectif d'avoir des mesures empiriques de l'insight, l'insight clinique a été considéré comme une reconnaissance, une capacité d'attribution, une solidité de la croyance. Progressivement, deux types d’évaluation ont été réalisés : catégorielle et dimensionnelle.

\subsubsection{L'exploration de l'insight selon une approche catégorielle}

Les premières évaluations de l’insight ont été réalisées de manière catégorielle, c’està-dire en tout ou rien, évaluant un insight partiellement présent ou partiellement absent. Cette évaluation peut se faire en hétéro-évaluation par un seul item, par exemple item G12 de l'échelle de la PANS (schizophrénie) [17], item 17 de l'échelle d'Hamilton (dépression) [17], item 11 de la Y-BOCS (trouble obsessionnel compulsif : TOC) [12;17], ou les items 104 et 105 dans le PSE (Present State Examination) [17]. Les mesures catégorielles sont utiles pour permettre des comparaisons entre individus sur des caractéristiques définies de manière large, mais comportent deux inconvénients majeurs, le premier étant la difficulté de bien distinguer les différentes catégories, et le second, ce qui est possible de déduire de ces descriptions catégorielles.

\subsubsection{L'exploration de l'insight selon une approche dimensionnelle}

L’évaluation dimensionnelle de l'insight a été proposée pour saisir quantitativement et avec plus de détails certaines des composantes explicitement définies de l'insight. McEvoy et al. en 1989 ont été les premiers à développer un questionnaire permettant d'évaluer l'insight : ITAQ (Insight and Treatment Attitude Questionnaire) chez les patients souffrant d'une schizophrénie [20]. Ce questionnaire est composé de 11 items, le score peut aller de 0 (pas d'insight) à 22 (insight maximal). Il est basé sur deux aspects : premièrement, l'insight est 
défini comme l'attitude du patient face à son traitement et à l'hospitalisation. Deuxièmement, les scores d'insight sont explicitement basés sur l'idée que le patient est en accord avec le professionnel de santé sur ces attitudes. En conséquence, le concept empirique d'insight exploré par cette mesure insiste davantage sur les visions concordantes qu'ont les patients de leur prise en charge que sur une quelconque compréhension détaillée de leur expérience morbide.

Une conceptualisation plus large de l'insight a été proposée par Greenfeld et al. en 1989 [in 17]. Ces auteurs proposent une évaluation qualitative de l’insight selon cinq dimensions - conscience : a) de la maladie, b) des symptômes, c) de la nécessité d’un traitement, d) de l'étiologie de la maladie, e) de la susceptibilité de rechuter. Ce concept plus large est davantage centré sur la compréhension des patients de ce qui leur arrive et du sens qu'ils donnent à leur expérience pathologique. Cependant, de manière pragmatique l'absence de score, car il s'agit d'évaluation qualitative, rend difficile la comparaison des groupes de patients.

La nécessité de mesurer l'insight avec obtention d'un ou des scores a conduit les chercheurs à la création des outils de mesures basée sur le modèle multidimensionnel de l'insight. Par exemple deux des échelles basées sur ce modèle sont : SAI (Schedule for the Assessment of Insight) proposée par David en 1990 [6], SUMD (The Scale to assess Unawareness in Mental Disorder) proposée par Amador et al. en 1991 [1,22,23]. Chez ces auteurs, l'accent est mis sur la transposition des modèles en mesures empiriques structurées évaluant l'insight quantitativement.

David, en se basant sur les travaux de Lewis, propose une définition tridimensionnelle de l'insight chez les patients souffrant d'une psychose : a) reconnaissance de la maladie mentale b) compliance au traitement c) capacité à reconnaître les événements psychotiques (idées délirantes et hallucinations) comme pathologiques. L’échelle SAI comporte 11 items cotés chacun de 0 (pas de conscience) à 4 (conscience complète), le score total peut aller de 0 à 14. Cette conception spécifique et plus étroite de l'insight le considère comme une reconnaissance (de la maladie et la nécessité d’un traitement) et un jugement (de la nature morbide des idées délirantes et hallucinations). Certains auteurs comme Birchwood [in 13], considèrent que la passation des échelles d'hétéro-évaluation de l'insight est chronophage avec des résultats difficilement reproductibles. Ainsi, en se fondant sur le concept tridimensionnel de l'insight proposé par David, Birchwood et al. proposent en 1994 une échelle d'autoévaluation de l'insight : Insight Scale. Cette échelle est constituée de huit items, avec un score pouvant aller de 0 à 4 pour chaque dimension. 
Amador et al. en 1991 [1], en se basant sur une analyse de la diversité des utilisations du terme d'insight et des termes apparentés dans la littérature, proposent de définir l’insight de manière multidimensionnelle - la conscience : a) de la maladie, b) des symptômes c) de la nécessité d’un traitement d) des conséquences sociale de la maladie, e) la capacité du patient à attribuer une cause à la maladie et/ou aux symptômes et à leurs conséquences, f) la capacité de rapporter l'image de Soi, g) les stratégies psychologiques de défense. La SUMD (Scale to assess Unawareness in Mental Disorder), issue de cette théorie, comporte 20 items ; les 3 premiers sont des items généraux et les 17 autres évaluent les symptômes spécifiques dans les pathologies psychotiques. Cette échelle peut être utilisée de manière partielle. Dans cette conception, la définition de l'insight apparaît plus large et plus détaillée, car elle incorpore dans une approche exhaustive la compréhension par le sujet de son vécu, le jugement porté par lui sur ce vécu, et des conceptions relatives de manière plus large à la compréhension du Soi et des processus psychologiques du Soi. Cependant, Amador et Strauss (1993) [2] reconnaissent les difficultés pratiques qu'il y a à passer d'une construction si large et si complexe à une mesure empirique de l'insight. Ainsi, ils ont préféré le réduire à un concept comprenant deux éléments importants : a) conscience de la maladie et b) capacité d'attribuer une cause à la maladie.

Une autre conceptualisation de l’insight est sa considération comme « solidité de la croyance » («strength of beliefs » en anglais) proposée par Eisen et al. 1988 [8]. BABS (Brown Assessment of Beliefs Scale) est issue de cette théorie et plus souvent utilisée dans les troubles obsessionnels compulsifs, la dysmorphophobie, et plus rarement dans la schizophrénie. Elle est une échelle d'hétéro-évaluation comportant 6 items avec un score allant de 0 à 24. Dans cette définition, plus les croyances délirantes sont ancrées et plus le niveau de l'insight est faible. Cette conception étroite de l'insight ne permet pas d'évaluer qu'une dimension de l'insight. Combs et al. en 2006 ont proposé CDBS (Conviction of Delusional Beliefs Scale) qui est une transposition de ce modèle en échelle d'autoévaluation [4].

Une autre approche pour l'évaluation de l'insight est celle proposée par Carsky et al. en 1992 [5]. Ils conceptualisent l'insight comme la réaction du patient face à son hospitalisation et se basent sur une définition limitée du déni. Ils ont mis en place le questionnaire d'autoévaluation PEH (Patients' Experience of Hospitalization) qui permet d'évaluer l'insight chez les patients souffrant d'une schizophrénie et hospitalisés. Ce questionnaire évalue l’incapacité du patient à reconnaître a) avoir une maladie ou que la maladie a un nom ou une cause, b) avoir besoin d'hospitalisation et (3) l'impact de la maladie 
sur le plan personnel. Cet instrument contient 18 items (chaque item est coté sur 4 points). Plus le score total est élevé, plus le déni est important. Cette échelle reflète de manière prédominante les jugements des patients sur le fait d'être hospitalisé. Une version adaptée de PEH aux patients suivis en ambulatoire a été proposée par Marks et al. (2000) [19]. Cette version, qui est toujours une autoévaluation, porte le nom de SAIQ (Self-Appraisal of Illness Questionnaire) et comprend 17 items.

Une conceptualisation dimensionnelle large de l’insight a été proposée par Marková et Berrios en 1992 [15]. Dans ce concept, l’insight est défini comme la conscience de changements du Soi (et de la manière dont cela affecte ses perceptions et interactions avec le monde environnant) en relation avec l'état pathologique affectant le patient. À partir de ce concept, une échelle d'autoévaluation de l'insight IPQ (Insight in Psychosis Questionnaire) a été mise en place et validée chez des patients psychotiques. Elle comporte 30 questions avec une cotation dichotomique (d'accord/pas d'accord) [16].

\subsection{Insight cognitif}

Beck introduit en 2004 [3] le concept de l’insight cognitif, qu’il définit comme la capacité de la conscience de présenter des distorsions cognitives et de faire des interprétations erronées [in 25 pour une revue complète]. Il considère que quatre aspects de l'insight cognitif peuvent être touchés dans la psychose : a) altération de la capacité d'être objectif sur les expériences délirantes et les distorsions cognitives, b) incapacité de mettre ces expériences en perspective, c) incapacité de corriger les informations venant des autres, d) l'excès de confidence dans les jugements délirants. Cette théorisation a donné lieu à la mise en place d’une échelle d'autoévaluation de l'insight : la BCIS (Beck Cognitive Insight Scale) [3]. Elle est composée de 15 items répartis en deux sous-échelles: la réflexion sur soi "SelfReflectiveness » et la certitude dans cette réflexion "Self-Certainty ». Dans cette conception étroite de l'insight on peut se demander à quel point une autoévaluation peut mesurer l'insight cognitif.

Une hétéro-évaluation de l'insight cognitif a été proposée par Medalia et al. en 2008 [21]. Ces auteurs, en se basant sur le modèle conceptuel de la SUMD, définissent l'insight cognitif comme la capacité du patient à reconnaître ses déficits cognitifs et à leur attribuer une cause. Ils ont proposé MIC-CR (Measure of Insight into Cognition-Clinician Rated), qui permet d'évaluer trois dimensions cognitives : mémoire, fonctions exécutives et attention. Cette échelle comporte 12 items, coté de 1 (insight total) à 5 (insight faible). Cette équipe a 
démontré que la mémoire joue un rôle peu important dans l'insight chez les patients souffrant d’une schizophrénie.

L’équipe de Poitiers (Gil et al. en 2001 [10]) a proposé une conceptualisation encore plus large et multidimensionnelle de l'insight en rapport avec la mémoire. Dans cette définition, basée sur les travaux de William James, l’insight est défini comme englobant tout un éventail de perceptions concernant l'identité propre, la conscience du corps, des processus mentaux (mémoire, autobiographie, pensées) et des jugements moraux. Une échelle d’hétéroévaluation dans la maladie d’Alzheimer a été mise en place [9].

\subsection{Insight comme une sensation somesthésique et sa localisation cérébrale}

Dans le but d'expliquer un faible insight par un dysfonctionnement cérébral, Lysaker et al. ont proposé en 1994 de définir l'insight comme l'anosognosie [14]. Dans cette définition, un faible insight est défini par la difficulté du patient à percevoir et à construire une représentation correcte de sa maladie. Ils ont utilisé des tests neuropsychologiques pour expliquer l'implication du lobe frontal dans l'insight chez les patients souffrant d'une schizophrénie. Cependant, les techniques d’imagerie cérébrale ont permis de trouver une corrélation entre un faible insight et d'autres parties du cerveau que le lobe frontal. Certains auteurs, en utilisant l'analyse morphométrique (voxel-based morphometry), rapportent une diminution de volume de la matière grise du cortex frontal, temporal, pariétal, cingulaire en rapport avec un faible insight, alors que d'autres études ne confirment pas cette idée (pour une revue complète voir [22] et Orliac et al. dans ce même numéro). Dans l'idée de trouver une localisation cérébrale à l'insight, l'équipe de Bechara en 2009 [11] propose de définir l’insight comme la capacité de reconnaître ses sensations somesthésiques chez les patients souffrant d'une addiction. Ces auteurs ont montré que la lésion du cortex insulaire chez les patients tabagiques entraîne une diminution de la capacité de reconnaissance du manque de la nicotine. Ainsi, l'arrêt du tabac chez ces patients serait plus simple que chez les patients souffrant d’une lésion cérébrale localisée ailleurs qu’au niveau du cortex cingulaire. Cette vision étroite du concept de l'insight permet une évaluation unidimensionnelle de l'insight, mais en quoi la capacité de se ressentir peut-elle représenter un concept si vaste ? Une nouvelle conceptualisation de l'insight somesthésique est proposée par l'équipe de Poitiers, Belin et al. (à lire dans ce même numéro).

L'existence de ces différentes échelles de mesures reflète différentes manières de conceptualiser l’insight. Les variations de méthode d'évaluation d’insight, autoévaluation 
versus hétéro-évaluation, retentissent sur le type d’insight qui est évalué. Par exemple, lors d’une hétéro-évaluation, si les mesures par un clinicien sont biaisées par son vécu, son jugement, les mesures lors des autoévaluations ont à l'inverse le défaut de ne pas permettre une interprétation aussi riche.

\subsection{Comment comprendre l'insight?}

La lecture des paragraphes précédents montre que les études empiriques n’arrivent pas à saisir l'insight dans sa globalité. Chacun y va de sa définition et de son échelle. Il semble que les difficultés rencontrées dans le domaine de la recherche empirique soient secondaires à la complexité du concept même d'insight. Il faut donc clarifier ce concept et les aspects que nous explorons par les évaluations cliniques. Dans cet objectif, il est important dans un premier temps de faire une distinction entre le concept et le phénomène d'insight et dans un second temps d'examiner les facteurs qui influencent le phénomène d'insight.

Le concept d'insight est une structure théorique qui nous aide à définir l'insight dans sa globalité, en identifiant les différents éléments qui le constituent. Il n’est pas réaliste d'essayer de capturer ce concept vaste dans une simple évaluation clinique. Chaque évaluation clinique ne touche qu'un des aspects du concept.

Le phénomène de l’insight se réfère à cet aspect de l’insight qui est recherché sur le plan clinique ou dans les études empiriques. Il représente seulement une partie du concept théorique de l’insight (figure 1).

Dans les études empiriques, le phénomène d'insight devient l'objet de l'étude, ce qui nous conduit au second point de clarification, à savoir, quels sont les facteurs qui influencent le phénomène clinique de l’insight (voir figure 1).

Premièrement, le phénomène sera influencé par le concept d’insight utilisé par le clinicien ou le chercheur. Comme mentionné ci-dessus, nous utilisons différents concepts d'insight, contenant des éléments différents, allant d'une vision étroite à une vision large de ce concept. Ainsi, les cliniciens ou chercheurs, selon le concept qu'ils utilisent, mettent en évidence, dans les études empiriques, différents aspects de l’insight.

Deuxièmement, l'échelle de mesure utilisée pour évaluer l'insight permettra de déterminer le phénomène qui est recherché. Les mesures de l’insight varient en fonction de leur contenu, c’est-à-dire évaluent les différents aspects des connaissances des patients concernant leur condition. Par exemple, certaines mesures pourraient être axées de manière générale sur les jugements des patients portant sur la cause de leur trouble. D’autres 
pourraient se concentrer davantage sur des évaluations précises et détaillées de la capacité des patients à décrire des symptômes particuliers ou des comportements. En outre, les mesures d'insight varient en fonction de leurs structures, à savoir que certaines mesures sont issues de l'autoévaluation, d'autres de l'hétéro-évaluation et d'autres encore de la méthode de l'écart de mesure, etc. Cela est important car les jugements des cliniciens, dans l'interaction avec les patients, contribuent à des degrés divers à l'élaboration du phénomène de l’insight.

Troisièmement, le phénomène d’insight est également influencé par l'« objet » d'évaluation d'insight. Insight est un terme relationnel qui signifie que nous ne pouvons le comprendre que s’il se porte sur quelque chose, que ce soit un état normal ou un état pathologique. Ainsi, on ne peut pas avoir d'insight sans qu'il y ait quelque chose sur lequel se porte l'insight. Et ce «quelque chose » a été appelé l'« objet » d'évaluation d’insight. En pratique clinique, il existe de nombreux «objets » d'évaluation d'insight, par exemple « symptômes mentaux », " maladie mentale », « dysfonctionnements neuropsychologiques », «troubles neurologiques », etc. Ces « objets» d'évaluation d’insight sont importants de différentes manières, car à leur tour ils influencent le phénomène de l'insight qui est recherché. La façon dont les objets d'évaluation d’insight influencent le phénomène de l'insight a été explorée ailleurs [17]. En bref, les objets peuvent être différents selon qu’il s’agit de a) différentes disciplines cliniques, b) différentes catégories sémantiques, c) et qu'ils se réfèrent aux différents types de choses.

Lorsque des « objets » d'évaluation d'insight se rapportent aux différentes disciplines cliniques, les phénomènes d’insight qui sont recherchés par rapport à ces objets seront structurés par la langue, l'histoire, les concepts et les hypothèses propres à cette discipline. Par exemple, le phénomène d’insight par rapport à un trouble mental sera structuré différemment du phénomène d’insight en rapport avec des troubles de mémoire ou du phénomène d’insight en rapport avec la motivation, et ce en raison des différents cadres conceptuels qui les soustendent en tant que discipline, à savoir respectivement la psychiatrie, la neuropsychologie et la psychanalyse.

Lorsque des « objets » d'évaluation d’insight appartiennent à différentes catégories sémantiques, alors le phénomène d’insight provoqué sera différent selon les catégories, lesquelles prennent en compte les différentes sortes de jugements qui pourraient être exigées par ces dernières. Par exemple, un «trouble mental » appartient à une catégorie sémantique différente d'un «dysfonctionnement neurologique ». Le premier est une construction qui s’appuie sur des jugements couvrant non seulement l'expérience mais aussi des changements plus larges intégrant les déterminants sociaux, individuels, culturels et politiques de cette 
construction. En revanche, « un dysfonctionnement neurologique » est une notion spécifique et relativement objective qui exige des jugements qui sont surtout concentrés sur un changement d'un état particulier. La nature et le type de jugements qui constituent les phénomènes d'insight dans chaque cas seront donc nécessairement très différents.

Lorsque des « objets » d'évaluation d'insight se réfèrent à différents types de choses, là encore, les phénomènes d'insight respectifs seront construits de manière différente. Par exemple, un « déficit neurologique » comme l’hémiplégie est très différent d’un "symptôme mental subjectif » comme les hallucinations. Le phénomène d'insight recherché par rapport à l'hémiplégie (pathologie objective) mettra l’accent très précisément sur la conscience de ce trouble et des jugements visant à le caractériser (par exemple la gravité, les effets, etc.), alors que le phénomène d’insight recherché en rapport avec les hallucinations aura une orientation complètement différente. Effectivement, par définition, la conscience des hallucinations est intrinsèque aux symptômes (par exemple, les patients ne seraient pas en mesure de dire s'ils entendent des voix, s’ils ne les reconnaissent pas). Ainsi, le phénomène d'insight dans ce cas se concentre sur une vision plus large, en incluant le sens que le patient donne à son expérience.

Au décours de cet article, on se rend compte que l'insight est un concept complexe et que sa considération en tant que symptôme pose un problème difficile à résoudre. Considérer l'insight en tant qu'état mental permet de comprendre qu'il s'agit d'un processus dynamique qui va varier en fonction des changements internes et externes. Les conséquences de l'insight en tant qu'état mental impliqueraient l'existence de certains aspects stables et résistants aux changements et d'autres aspects modifiables et variables dans le temps. En conséquence, l'insight en tant qu'état mental permet la construction d'une structure qui expliquerait ces changements et apporterait une compréhension des résultats contradictoires des études. On peut retenir que le choix d’une échelle psychométrique doit se faire en se posant trois questions : quel est le concept utilisé ? Quel est le phénomène d'insight recherché ? Quel est l'objet de l'insight?

\section{Conclusion}

L’insight est un concept complexe sans définition unitaire. La recherche empirique sur l'insight est entravée par des complexités propres au concept d’insight. Dans l'idée d'une compréhension des problèmes posés il faut distinguer le concept et le phénomène. Le 
phénomène d'insight reflète seulement certains aspects du concept d'insight. Les facteurs qui influencent le phénomène d'insight comportent: le concept d'insight, le type d'outils de mesure qui sont utilisés et l'objet de l'insight. Définir de tels facteurs est essentiel pour toute recherche empirique. Cette démarche aidera à délimiter le phénomène spécifique d'insight étudié. Pour une compréhension de l'insight, nous conseillons aux lecteurs de lire Insight en psychiatrie [17] et de regarder les deux congrès internationaux sur l'insight qui ont eu lieu à Poitiers en 2008 et 2009 (présentations et diapositives accessibles gratuitement) [24].

\section{Conflit d'intérêt : aucun}

Remerciement : cette étude a été soutenue par l’Unité de Recherche Clinique intersectorielle en psychiatrie du Centre Hospitalier Henri Laborit qui a obtenu un financement par l'Agence Régionale de la Santé de Poitou-Charentes. 


\section{Références}

[1] Amador XF, Strauss DH, Yale SA, Gorman JM. Awareness of illness in schizophrenia. Schizophr Bull 1991;17:113-32.

[2] Amador XF, Strauss DH. Poor insight in schizophrenia. Psychiatr Q 1993;64:305-18.

[3] Beck AT, Baruch E, Balter JM, et al. A new instrument for measuring insight: the Beck Cognitive Insight Scale. Schizophr Res 2004;68:319-29.

[4] Combs DR, Adams SD, Michael CO, et al. The conviction of delusional beliefs scale: reliability and validity. Schizophr Res 2006;86:80-8.

[5] Carsky M, Selzer MA, Terkelsen K, et al. The PEH. A questionnaire to assess acknowledgment of psychiatric illness. J Nerv Ment Dis 1992;180:458-64.

[6] David AS. To see ourselves as others see us. Aubrey Lewis's insight. Br J Psychiatry 1999;175:210-6.

[7] David AS. Insight and psychosis. Br J Psychiatry 1990;156:798-808.

[8] Eisen JL, Phillips KA, Baer L, et al. The Brown Assessment of Beliefs Scale: reliability and validity. Am J Psychiatry 1998;155:102-8.

[9] Fargeau MN, Jaafari N, Ragot S, et al. Alzheimer's disease and impairment of the Self. Conscious Cogn 2010;19:969-76.

[10] Gil R, Arroyo-Anllo EM, Ingrand P, et al. Self-consciousness and Alzheimer's disease. Acta Neurol Scand 2001.

[11] Goldstein RZ, Craig AD, Bechara A, et al. The neurocircuitry of impaired insight in drug addiction. Trends Cogn Sci 2009;13:372-80.

[12] Jaafari N, Aouizerate B, Tignol J, et al. The Relationship between Insight and Uncertainty in Obsessive-Compulsive Disorder. Psychopathology 2011;44:272-6.

[13] Jaafari N, Daniel ML, Paillot C. Échelle d'autoévaluation de l'insight de Birchwood. Évaluation clinique standardisée en psychiatrie, par Guelfi. Paris: Pierre Fabre édition; 2011.

[14] Lysaker P, Bell M. Insight and cognitive impairment in schizophrenia. Performance on repeated administrations of the Wisconsin Card Sorting Test. J Nerv Ment Dis 1994;182:65660.

[15] Marková IS, Berrios GE. The assessment of insight in clinical psychiatry: a new scale. Acta Psychiatr Scand 1992;86:159-64.

[16] Marková IS, Roberts KH, Gallagher C, et al. Assessment of insight in psychosis: a restandardization of a new scale. Psychiatry Research 2003;119:81-8.

[17] Marková IS. Insight en psychiatrie. Traduction Jaafari et al. Paris: Doin édition; 2009. 
[18] Marková IS, Jaafari N, Berrios GE. Insight and obsessive-compulsive disorder: a conceptual analysis. Psychopathology 2009;42:277-82.

[19] Marks KA, Fastenau PS, Lysaker PH, et al. Self-Appraisal of Illness Questionnaire (SAIQ): relationship to researcher-rated insight and neuropsychological function in schizophrenia. Schizophr Res 2000;45:203-11.

[20] McEvoy JP, Apperson LJ, Appelbaum PS, et al. Insight in schizophrenia. Its relationship to acute psychopathology. J Nerv Ment Dis 1989;177:43-7.

[21] Medalia A, Thysen J. Insight into neurocognitive dysfunction in schizophrenia. Schizophr Bull 2008;34:1221-30.

[22] Morgan KD, Dazzan P, Morgan C, et al. Insight, grey matter and cognitive function in first-onset psychosis. Br J Psychiatry 2010;197:141-8.

[23] Paillot C, Ingrand P, Millet B, et al. Insight Study Group. French translation and validation of the Scale to assess Unawareness of Mental Disorder (SUMD) in patients with schizophrenics. Encéphale. 2010;36:472-7.

[24] Premier et deuxième congrès international d'insight en psychiatrie et en neurologie à Poitiers (accès gratuit :http://uptv.univpoitiers.fr/web/canal/61/theme/24/manif/183/index.html, $\quad$ http://uptv.univpoitiers.fr/web/canal/61/theme/24/manif/221/index.html

[25] Riggs SE, Grant PM, Perivoliotis D, et al. Assessment of Cognitive Insight: A Qualitative Review. Schizophr Bull 2010 Aug 6.

Figure 1 : schématisation de l’insight 


\section{Schématisation de l’insight}

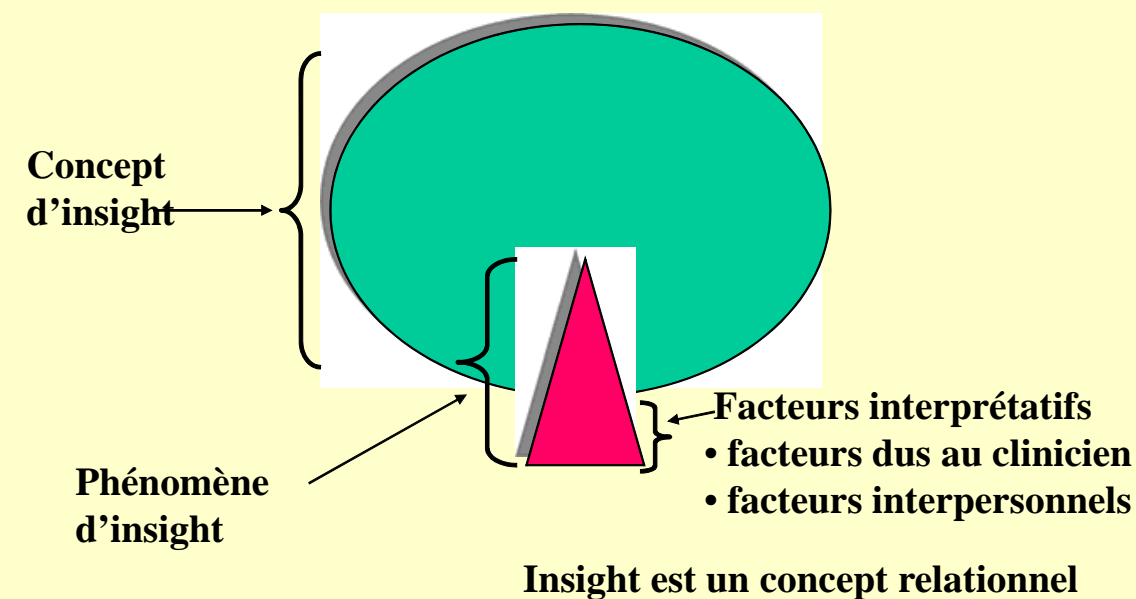

Si nous schématisons l'insight comme un vaste ensemble (bulle), le phénomène d’insight peut être représenté par une partie de cet ensemble (triangle)_qui ne décrit qu’un seul de ses aspects.

\section{Discussion}

$\operatorname{Pr}$ A. Pelissolo - Merci beaucoup au Dr Jaafari pour avoir exposé tous les éléments du problème en ce début de séance, de manière exhaustive et didactique, et je signale sa traduction du livre de Markova, L'Insight en psychiatrie (Paris, Doin, 2009). La question qui me vient en premier lieu est celle de l'existence ou non d'un facteur général d'insight, ou plutôt de plusieurs dimensions indépendantes les unes des autres. L'exemple qui me fait favoriser la seconde hypothèse est celui des troubles anxieux, TOC ou dysmorphophobie en particulier, dans laquelle on peut avoir à la fois une conscience très douloureuse donc très vive d'un trouble (égodystonie) et un trouble du jugement sur certaines croyances ou pensées (distorsions cognitives, pensées surévaluées). 
$\operatorname{Pr}$ M. Laxenaire - J'aurais deux courtes questions. Pour la première, vous avez dit qu'il n’y avait pas d'équivalent français d'insight, mais que pensez-vous du terme « introspection »?

La deuxième concerne l'anosognosie: vous avez défini l'insight comme une connaissance, or l'anosognosie est une « méconnaissance », on ne peut donc assimiler l'une à l'autre.

Dr P. Moutin - Vous avez insisté, dans les interprétations multiples de l’insight, sur la conscience de soi et de la prise de conscience de la maladie et de ses conséquences, avec les applications psychothérapeutiques possibles.

Mais d'autres thérapies ont été proposées, sans faire référence précise à l’insight, à partir de la conscience de soi. C'est le cas, en particulier, du psychiatre cognitiviste Zindel Segal qui, avec deux collègues anglais, à, en 1993, mis au point une «thérapie cognitive basée sur la pleine conscience », où la technique principale est la méditation. Ne faudrait-il pas aussi en parler dans cette réunion, d'autant plus que cette théorie a eu beaucoup d’applications (Frédérique Rosenfeld, Méditer, c’est se soigner, éd. Les Arènes) ?

Réponse du Rapporteur - Je voudrais remercier le Pr Pelissolo pour ses propos sympathiques. Effectivement, l'insight peut être considéré comme un concept multidimensionnel avec des dimensions indépendantes. Il n’existe pas un seul facteur général. On peut avoir l'insight d'un phénomène particulier avec ou sans capacité de jugement

Au Pr M. Laxenaire - Je pense que l'on ne peut pas traduire l'insight par les termes « introspection» ou «anosognosie». L’introspection comme d'ailleurs le déni ont une connotation analytique et renvoient à la notion de l'inconscient. L'anosognosie a une connotation neuropsychologique et renvoie à l'incapacité du patient de reconnaître son hémicorps controlatéral à la lésion cérébrale. Or l’insight, comme je l’ai dit, est un concept plus large que ces termes ne pourraient pas représenter.

Au Dr P. Moutin - Il existe plusieurs types de psychothérapie avec une implication directe ou indirecte de l’insight. Il faudra une autre séance thématique de la Société MédicoPsychologique pour en parler. Le sujet est vaste et nous avons dû faire des choix, mais j'espère qu'on pourra organiser très rapidement une nouvelle journée sur les méthodes de psychothérapie et l’insight. 